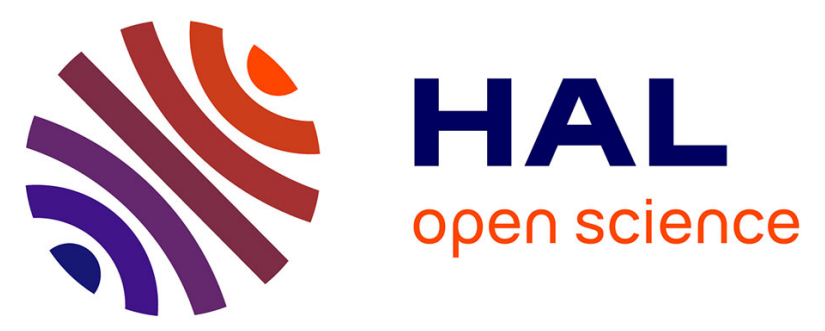

\title{
Modeling and design of passive electric networks interconnecting piezoelectric transducers for distributed vibration control
}

\author{
Maurizio Porfiri, Francesco Dell'Isola, Ezio Santini
}

\section{> To cite this version:}

Maurizio Porfiri, Francesco Dell'Isola, Ezio Santini. Modeling and design of passive electric networks interconnecting piezoelectric transducers for distributed vibration control. International Journal of Applied Electromagnetics and Mechanics, 2005, pp.19. hal-00509102

\section{HAL Id: hal-00509102 \\ https://hal.science/hal-00509102}

Submitted on 10 Aug 2010

HAL is a multi-disciplinary open access archive for the deposit and dissemination of scientific research documents, whether they are published or not. The documents may come from teaching and research institutions in France or abroad, or from public or private research centers.
L'archive ouverte pluridisciplinaire HAL, est destinée au dépôt et à la diffusion de documents scientifiques de niveau recherche, publiés ou non, émanant des établissements d'enseignement et de recherche français ou étrangers, des laboratoires publics ou privés. 


\title{
Modeling and design of passive electric networks interconnecting piezoelectric transducers for distributed vibration control
}

\author{
M. Porfiri ${ }^{\mathrm{a}, \mathrm{b}}$, F. dell'Isola ${ }^{\mathrm{a}, *}$ and E. Santini ${ }^{\mathrm{c}}$ \\ ${ }^{a}$ Dip. di Ing. Strutturale\& Geotecnica, "La Sapienza", 00184 Rome, Italy \\ ${ }^{\mathrm{b}}$ Engineering Science and Mechanics Department, Virginia Tech, Blacksburg, VA 24061-0219, USA \\ ${ }^{c}$ Dip. di Ing. Elettrica, "La Sapienza”, 00184 Rome, Italy
}

\begin{abstract}
In the literature collocated or distributed arrays of piezeoelectric patches are employed to actively control structural vibrations. In the present work in order to damp beam vibrations a completely passive electric controller is designed, exploiting distributed piezoelectric transduction. In this way a simultaneous multimodal vibration suppression is obtained. The optimal electric network (interconnecting the piezoelectric transducers) is synthesized as a finite differences approximation of the derived distributed (infinite dimensional) optimal controller. A prototype of the proposed novel smart structure (Piezo-ElectroMechanical beam) is designed, allowing for appreciating its technical feasibility and effectiveness.
\end{abstract}

\section{Introduction}

The recent technological developments in the production of piezoelectric transducers, and the relevant consumers' attention towards the suppression of structural vibrations, increased the research efforts in their effective exploitation in control systems for actual engineering structures. An efficient control of structural vibrations leads to other benefits, such as the precision in mechanisms manoeuvres, the reduced fatigue loads, the reliability and durability of machineries. These were the main reasons to attract the interest of both mechanical and aerospace industries in this topic.

The aim of this paper is to prove that simultaneous multimodal beam vibration suppression is technically feasible, when exploiting purely passive electric networks and available piezoelectric transducers. Indeed the authors prove that multimodal damping of mechanical vibrations by means of truly passive electric circuits can be obtained by uniformly distributing piezoelectric transducers on the host structure and suitably designing an optimal interconnecting electric passive network (the resulting smart structure is called Piezo-ElectroMechanical structure, PEM for brevity). This concept seems an interesting development of the method of "piezoelectric shunting" proposed by Hagood and von Flotow in [1] for single mode and extended by some other authors to multimodal control ([2] and [3]). Indeed, the method here presented is based on the shunting of an array of distributed piezoelectric transducers with a multiterminal terminals passive electric network: more precisely, instead of coupling each piezoelectric transducer to a single (eventually multifrequency) electric resonator, the whole set of transducers is coupled to a

\footnotetext{
${ }^{*}$ Corresponding author. Tel.: +39 0644585 297; Fax: +39 064884 852; E-mail: Francesco.Dellisola@ @niroma1.it.
} 


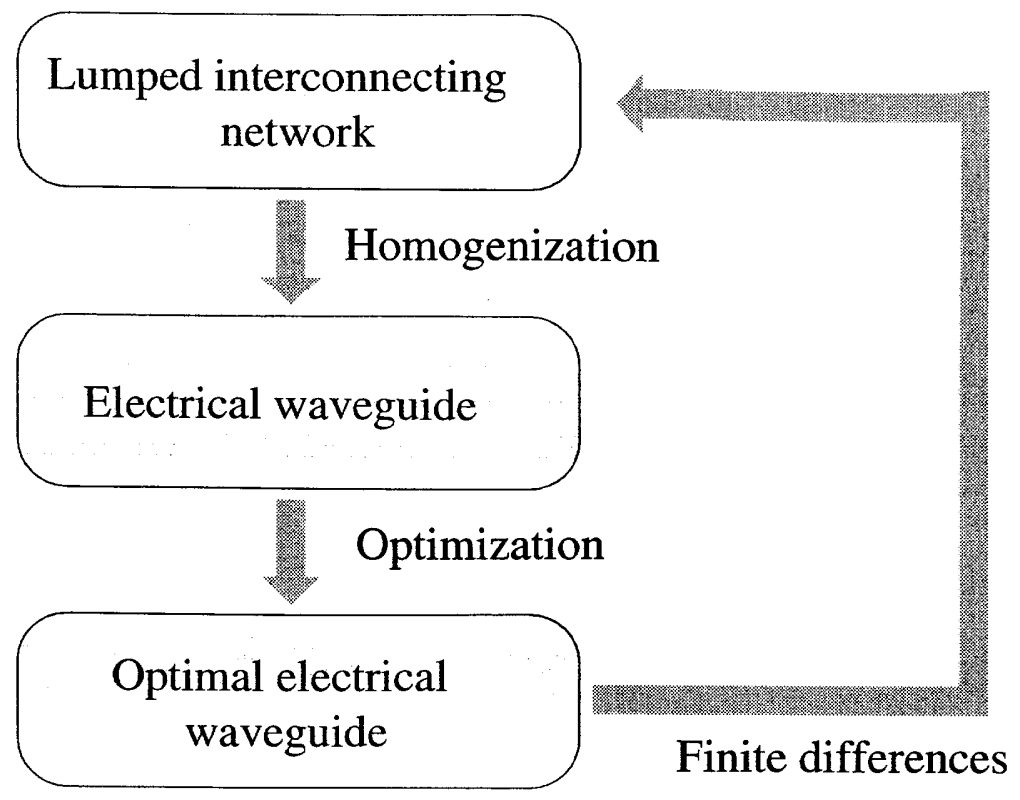

Fig. 1. Flow chart describing the adopted methodology for the synthesis of the electrical interconnecting network.

distributed electric network. It has to be remarked that the presented electric dynamic controller evolves with second order differential equations in time gyroscopically coupled to those governing the evolution of the mechanical system to be controlled, and therefore the concept introduced here differs from that studied in [4-6] and [7]. Indeed, in the quoted papers the distributed array of piezoelectric transducers is actively driven by a voltage field, which is the output of a suitable feed-back loop (in this way one gets a smart structure which is in general active). Instead, the piezoelectric controller here introduced is completely passive, so that PEM structures are passive.

In Section 2 a refined (microscopic) model for PEM beams is introduced, in which the lumped nature of the electric network, the material discontinuities introduced by the piezoelectric laminae and the localization of piezoelectric applied couples are accounted for.

In Section 3 the homogenized model is deduced from the previously introduced refined model. Such a homogenized model:

1. is valid when the number of transducers positioned on the host structure is sufficiently large,

2. is more handleable when optimizing distributed controllers,

3. is sufficiently detailed to suggest design criteria for truly lumped PEM beams.

In Section 4, the modal analysis of homogenized PEM beams is addressed, without specifying completely the evolution equations of the electric controller, which is assumed to belong to a rather wide class of (local) differential controllers.

In Section 5, the pole placement technique is exploited to determine - in the class previously specified - the optimal passive electric controller, which establishes a multimodal critical damping. Moreover, by means of a finite differences scheme and by exploiting the results in [8] and [9], a lumped electric circuit representing a finite dimensional approximation of the optimal homogenized (infinite dimensional) controller is presented. The methodology is summarized in Fig. 1.

In Section 6, the set of electromechanical mode shapes and modal frequencies of the optimally controlled PEM beam are determined. 


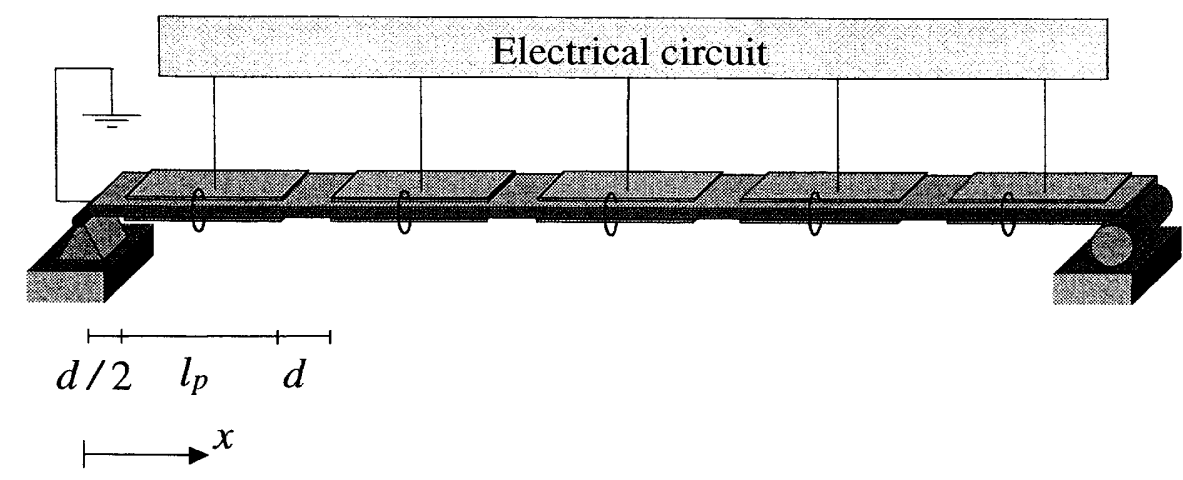

Fig. 2. Geometry of the problem.

In Section 7, the theoretical results previously established are exploited to start the design of a prototype of a PEM beam. The limits of the homogenized models are analyzed and the need of a more refined model is established. This last model will necessarily account for the lumped nature of the electric controller and will be studied in future works.

\section{Refined model for PEM beams}

We consider a host beam of length $l$, width $w$ and thickness $h$ on which an array of $N$ periodically distributed bender (see e.g. [8]) piezoelectric transducers is positioned ${ }^{1}$ as shown in Fig. 2 . The length of the transducers is assumed to be equal to $l_{p}$, while the width is assumed to be equal to that of the beam; $d$ denotes the distance between the adjacent patches.

These transducers will be interconnected by an electrical network which will be synthesized in order to accomplish given optimality conditions on the mechanical vibration decay.

By introducing the set of nodes $\left\{x_{i}\right\}_{i=1, \ldots N}$, representing the geometrical centers of the transducers, and defined by:

$$
x_{i}=\left(l_{p}+d\right)\left(i-\frac{1}{2}\right) \quad i=1, \ldots N,
$$

the bending moment $M$ at the section labelled by the abscissa $x$, over the beam span, may be expressed as the sum of mechanical and piezoelectric contributions as follows [8]:

$$
\begin{aligned}
M(x, t)= & {\left[E_{b} I_{b}+k_{m m} \sum_{i=1}^{N}\left(\operatorname{RECT}_{l_{p}}\left(x-x_{i}\right)\right)\right] u^{\prime \prime}(x, t) } \\
& +k_{m e} \sum_{i=1}^{N}\left(\operatorname{RECT}_{l_{p}}\left(x-x_{i}\right) \dot{\psi}_{i}(t)\right)
\end{aligned}
$$

where:

$$
\operatorname{RECT}_{l_{p}}\left(x-x_{i}\right)=\mathrm{H}\left(x-x_{i}-\frac{l_{p}}{2}\right)-\mathrm{H}\left(x-x_{i}+\frac{l_{p}}{2}\right)
$$

\footnotetext{
${ }^{1}$ The piezoceramic laminae are on both the beam surfaces. They are polarized in the transverse direction.
} 


$$
k_{m m}=\frac{h^{2} w \delta}{2 s_{11}^{E}}, \quad k_{m e}=\frac{h w d_{31}}{s_{11}^{E}}
$$

$\mathrm{H}$ is the Heaviside function, $\psi_{i}$ is the flux linkage ${ }^{2}$ of the $i$-th transducer measured with respect to a common reference ground for every transducer, $u$ is the beam deflection field, $E_{b}$ is the Young modulus of the material of the beam, $I_{b}$ is the beam section moment of inertia $\left(I_{b}=h^{3} w / 12\right), \delta$ is the transducers thickness ${ }^{3}, s_{11}^{E}$ is the piezoelectric mechanical compliance, $d_{31}$ is the piezoelectric coupling coefficient, $t$ denotes the time variable and superimposed dot and prime respectively mean time and space derivative. In the literature, see e.g. [10], several models of beams hosting piezoelectric transducers have been proposed leading to different estimations of the constants in Eq. (3b).

The distributed inertia can be accounted for by introducing the following constitutive equation for the applied load (external mechanical forcing is excluded):

$$
b_{T}(x, t)=-w h\left(\rho_{b}+2 \frac{\delta}{h} \sum_{i=1}^{N}\left(\operatorname{RECT}_{l_{p}}\left(x-x_{i}\right)\right) \rho_{p}\right) \ddot{u}(x, t),
$$

where $\rho_{b}$ and $\rho_{p}$ are respectively the mass density per unit volume of the beam and transducer materials.

The balance equations for the considered electrically excited vibrating beam yield:

$$
M(x, t)^{\prime \prime}-b_{T}(x, t)=0
$$

where $M(x, t)$ and $b_{T}(x, t)$ are given by Eqs (3) and (4) respectively.

Assuming, as in Fig. 1, the beam to be simply supported, the mechanical boundary conditions to be considered are:

$$
\left\{\begin{array}{l}
u(0, t)=u(l, t)=0 \\
M(0, t)=M(l, t)=0
\end{array} .\right.
$$

From a purely electrical point of view, the $i$-th piezoelectric bender transducer can be described as a capacitor in parallel connection with a "mechanically driven" current source, which injects into the electrical circuit the current $J_{i}$ driven by its mechanical time rate of deformation (see Fig. 3) [8]:

$$
J_{i}=k_{m e}\left(\dot{u}^{\prime}\left(x_{i}+l_{p} / 2, t\right)-\dot{u}^{\prime}\left(x_{i}-l_{p} / 2, t\right)\right) .
$$

The capacitance of each piezoelectric bender transducer can be estimated to be equal to [8]:

$$
k_{e e}=2 \frac{w\left(s_{11}^{E} \epsilon_{3}^{T}-d_{31}^{2}\right)}{s_{11}^{E} \delta} l_{p}
$$

The electrical system interconnecting the electrical terminals of the bender transducers is assumed to be a linear, time invariant, reciprocal, passive $N+1$ terminals network (see [11]) the admittance matrix $\left[Y^{e}\right]$ of which, in the Laplace domain, is given by:

$$
\left[Y^{e}\right]=\left[D^{e}\right]+\frac{1}{s}\left[K^{e}\right]
$$

\footnotetext{
${ }^{2}$ The flux linkage $\psi_{i}$ represents the time integral of the voltage $V_{i}$ of the $i$-th transducer measured with respect to the common ground, i.e. $\dot{\psi}_{i} \equiv V_{i}$.

${ }^{3}$ The transducers thickness $\delta$ is assumed to be much smaller than the beam thickness.
} 
appears. Equation (10a) states that the effective compliance is equal to the weighted sum of the compliances of the homogeneous beam and of the beam covered by the piezoelectric elements. Equation (10b) states that the ratio of the effective coupling to the effective stiffness is equal to the ratio of the piezoelectric coupling times the covering factor to the stiffness of the beam covered by the piezoelectric patches. The average of the above introduced flux linkage field $\psi$ on the region covered by the $i$-th transducer is equal to the the flux linkage $\psi_{i}$. Similarly the applied load Eq. (4) may be written as:

$$
b_{T}=-\rho_{\mathrm{hom}} \ddot{u},
$$

where the homogenized linear mass density is given by the weighted sum of the linear mass densities of the homogeneous beam and of the beam covered by the piezoelectric patches, i.e.

$$
\rho_{\mathrm{hom}}=w h\left(\rho_{b}+2 c_{f} \frac{\delta}{h} \rho_{p}\right)
$$

Thus the dimensionless form of the mechanical evolution equation becomes:

$$
\ddot{u}+\alpha^{4} u^{I V}+\beta_{e m}^{2} \dot{\psi}^{\prime \prime}=0, \quad\left\{\begin{array}{l}
\alpha^{4}=\frac{k_{\mathrm{hom}}}{l^{4} \rho_{\mathrm{hom}} \omega_{0}^{2}} \\
\beta_{e m}^{2}=\frac{g_{\mathrm{hom}} \psi_{0}}{l^{2} \rho_{\mathrm{hom}} \omega_{0} u_{0}}
\end{array},\right.
$$

where a characteristic radian frequency $\omega_{0}$, a characteristic length equal to the beam length $l$, a characteristic deflection $u_{0}$ and a characteristic flux linkage $\psi_{0}$ have been introduced. As this cannot cause misunderstanding, we have adopted the same symbols to denote both dimensional and dimensionless differential operators and kinematical descriptors. Within the homogenized model (see e.g. Eq. (9)) the mechanical boundary conditions Eq. (6) to be considered in the analysis of the simply supported PEM beam are:

$$
\left\{\begin{array}{l}
u(0, t)=u(1, t)=0 \\
k_{\mathrm{hom}} u^{\prime \prime}(0, t)+g_{\mathrm{hom}} \dot{\psi}(0, t)=k_{\mathrm{hom}} u^{\prime \prime}(1, t)+g_{\mathrm{hom}} \dot{\psi}(1, t)=0,
\end{array}\right.
$$

The mechanical modal properties (mode shapes $v_{m}$ 's and eigenvalues $\lambda_{m}$ 's) of the PEM beam when the piezoelectric transducers are short-circuited to ground are obtained by solving the eigenvalue problem

$$
v^{I V}=\lambda v
$$

where the boundary conditions are Eq. (13) with vanishing voltage. Therefore the mode shapes are

$$
v_{m}(x)=\sqrt{2} \sin (m \pi x), \quad m=1,2, \ldots,
$$

and the corresponding eigenvalues are given by

$$
\lambda_{m}=(m \pi)^{4}, \quad m=1,2, \ldots .
$$




\subsection{Homogenized circuit}

Similarly, when the number of transducers can be assumed to be sufficiently large, the electrical system can be described by a sole PDE which, expressed in terms of the dimensionless flux-linkage $\psi$, reads:

$$
\ddot{\psi}+\mathbf{K}[\psi]+\mathbf{D}[\dot{\psi}]-\beta_{m e}^{2} \dot{u}^{\prime \prime}=0, \quad \beta_{m e}^{2}=\frac{g_{\mathrm{hom}} u_{0}}{l^{2} \gamma_{\mathrm{hom}} \omega_{0} \psi_{0}},
$$

where the homogenized piezoelectric capacitance per unit length

$$
\gamma_{\mathrm{hom}}=\frac{c_{f} k_{e e}}{l_{p}}
$$

appears, together with the two linear operators $\mathbf{K}$ and $\mathbf{D}$ accounting for the electric shunting network. In the present work, it is assumed that $\mathbf{K}$ and $\mathbf{D}$ are spatial differential operators of the form

$$
\left\{\begin{array}{l}
\mathbf{K}[\psi]=\sum_{i=0}^{A}(-1)^{i} a_{2 i} \frac{d^{(2 i)} \psi}{d x^{(2 i)}} \\
\mathbf{D}[\dot{\psi}]=\sum_{i=0}^{B}(-1)^{i} b_{2 i} \frac{d^{(2 i)} \dot{\psi}}{d x^{(2 i)}}
\end{array},\right.
$$

where $2 A$ and $2 B$ indicate the order of the operators (being greater or equal than 2), and $a_{2 i}$ and $b_{2 i}$ are nonnegative real constants with $a_{2 A}$ and $b_{2 B}$ greater than zero. The domains of the operators, $\mathcal{D}(\mathbf{K})$ and $\mathcal{D}(\mathbf{D})$ are defined by

$$
\begin{array}{r}
\mathcal{D}(\mathbf{K})=\left\{f \in L^{2}: f, f^{\prime}, \ldots, f^{(2 A-1)}\right. \text { are absolutely continuous, } \\
\left.f^{(2 A)} \in L^{2}, f^{(2(i-1))}(0)=f^{(2(i-1))}(1)=0, i=1, \ldots A\right\},
\end{array}
$$

and

$$
\begin{array}{r}
\mathcal{D}(\mathbf{D})=\left\{f \in L^{2}: f, f^{\prime}, \ldots, f^{(2 B-1)}\right. \text { are absolutely continuous, } \\
\left.f^{(2 A)} \in L^{2}, f^{(2(i-1))}(0)=f^{(2(i-1))}(1)=0, i=1, \ldots B\right\} .
\end{array}
$$

The class of differential operators of the form Eq. (16) and the assumed boundary conditions have been considered for the following reasons:

1. the electrical system piezoelectrically coupled to the flexible structure does not introduce any spillover phenomenon among the mechanical vibration modes;

2. the synthesis of an electrical circuit governed by a discrete, finite differences ${ }^{4}$, form of these operators is very easy, exploiting the methods adopted in [8];

3. it includes some interesting interconnection schemes for piezoelectric arrays, developed in the vibration control literature $([12,13])$.

\footnotetext{
${ }^{4}$ The relationship between the operators $\mathbf{K}$ and $\mathbf{D}$ and the matrices $\left[K^{e}\right]$ and $\left[D^{e}\right]$ in Eq. (7) depends on the adopted discretization technique. Indeed different approximation techniques yield distinct residue matrices, and consequently distinct optimal circuits. In this paper we refer to finite differences symmetric schemes and expressions for $\left[K^{e}\right]$ and $\left[D^{e}\right]$ may be derived by the use of the techniques in [14].
} 
From the above assumptions, both the operators are self-adjoint and share the same electric mode shapes, which coincide with the mechanical mode shapes of the PEM beam with grounded piezoelectric elements given in Eq. (14). On the contrary, their eigenvalues may be different and depend on the constants $a_{2 i}$ and $b_{2 i}$. In particular, by substituting Eq. (14) into Eq. (16) we obtain

$$
\left\{\begin{array}{l}
\mathbf{K}\left[v_{m}\right]=\left(\sum_{i=0}^{A} a_{2 i}(m \pi)^{2 i}\right) v_{m} \\
\mathbf{D}\left[v_{m}\right]=\left(\sum_{i=0}^{B} b_{2 i}(m \pi)^{2 i}\right) v_{m}
\end{array}\right.
$$

therefore, the eigenvalues of the electric operators are

$$
\left\{\begin{array}{l}
P_{\mathbf{K}}(m)=\sum_{i=0}^{A} a_{2 i}(m \pi)^{2 i} \equiv \sum_{i=0}^{A} a_{2 i}\left(\lambda_{m}\right)^{i / 2} \\
P_{\mathbf{D}}(m)=\sum_{i=0}^{B} b_{2 i}(m \pi)^{2 i} \equiv \sum_{i=0}^{A} a_{2 i}\left(\lambda_{m}\right)^{i / 2}
\end{array}\right.
$$

where the eigenvalues $\lambda_{m}$ 's in Eq. (15) have been used. We remark that the proposed differential operators are linear combinations of powers of the fourth derivative operator (see e.g. [15]) as follows:

$$
\left\{\begin{array}{l}
\mathbf{K}[\cdot]=a_{0}\left((\cdot)^{I V}\right)^{0}+a_{2}\left((\cdot)^{I V}\right)^{1 / 2}+a_{4}\left((\cdot)^{I V}\right)^{1}+\ldots+a_{2 A}\left((\cdot)^{I V}\right)^{A / 2} \\
\mathbf{D}[\cdot]=b_{0}\left((\cdot)^{I V}\right)^{0}+b_{2}\left((\cdot)^{I V}\right)^{1 / 2}+b_{4}\left((\cdot)^{I V}\right)^{1}+\ldots+b_{2 B}\left((\cdot)^{I V}\right)^{B / 2}
\end{array}\right.
$$

As an example, we notice that when

$$
A=B=1, \quad a_{0}=b_{0}=0,
$$

the electric operators become

$$
\left\{\begin{array}{l}
\mathbf{K}[\cdot]=a_{2}\left((\cdot)^{I V}\right)^{1 / 2} \equiv-a_{2}(\cdot)^{\prime \prime} \\
\mathbf{D}[\cdot]=b_{2}\left((\cdot)^{I V}\right)^{1 / 2} \equiv-b_{2}(\cdot)^{\prime \prime}
\end{array} .\right.
$$

In this instance, each piezoelectric transducer is connected to the adjacent one by a floating inductiveresitive impedance, providing a second order transmission line piezoelectrically coupled to the vibrating structure ([12] and [13]). In what follows, we will see that when only a single mode suppression is needed. This techniques is extremely efficient and (see e.g. [16]) allows a substantial reduction of the needed nominal inductance with respect to the classical piezoelectric shunting of [1].

\subsection{Homogenized PEM beam}

The dimensionless evolution electromechanical equations of the resulting PEM beam are:

$$
\left\{\begin{array}{l}
\ddot{u}+\alpha^{4} u^{I V}+\beta^{2} \dot{\psi}^{\prime \prime}=0 \\
\ddot{\psi}+\mathbf{K}[\psi]+\mathbf{D}[\dot{\psi}]-\beta^{2} \dot{u}^{\prime \prime}=0
\end{array} ; \quad\left\{\begin{array}{l}
\alpha^{4}=\frac{k_{\mathrm{hom}}}{l^{4} \omega_{0}^{2} \rho_{\mathrm{hom}}} \\
\beta^{2}=g_{\mathrm{hom}} \frac{1}{l^{2} \omega_{0}} \sqrt{\frac{1}{\gamma_{\mathrm{hom}} \rho_{\mathrm{hom}}}},
\end{array}\right.\right.
$$


where, in order to preserve the form of a gyroscopic coupling, the characteristic flux linkage and displacement are chosen to satisfy the following relation:

$$
\sqrt{\frac{\gamma_{\text {hom }}}{\rho_{\text {hom }}}}=\frac{u_{0}}{\psi_{0}}
$$

The choice of the electrical boundary conditions implied by the definition of the domains of the electrical operators decouples the mechanical boundary conditions from the electrical system. Indeed the voltage field vanishes at the beam ends, and the boundary conditions Eq. (13) reduce to those of the PEM beam when all the transducers are grounded.

\section{Modal analysis of homogenized PEM beams}

In this Section a modal analysis for the simply-supported PEM beam, based upon the Galerkin method, is performed.

The set of basis functions chosen to represent the deflection $u(x, t)$ and the flux linkage $\psi(x, t)$ of the PEM beam is constituted by the eigenfunctions $v_{m}(x)$ specified in Eq. (14) of the fourth derivative determining the mode shapes for both the electrical and mechanical distributed systems Eq. (18).

Consequently, it is useful to consider a Fourier expansion for the solution of Eq. (18):

$$
\left[\begin{array}{l}
u(x, t) \\
\psi(x, t)
\end{array}\right]=\sum_{m=1}^{\infty}\left[\begin{array}{l}
p_{m}(t) \\
q_{m}(t)
\end{array}\right] v_{m}(x),
$$

in terms of the mechanical and electrical Fourier coefficients $p_{m}(t)$ and $q_{m}(t)$ of the expansion. With simple algebraic manipulations the following system of ODEs governing their time evolution, in which unitary dimensionless modal masses appear, is obtained:

$$
\left[\begin{array}{ll}
1 & 0 \\
0 & 1
\end{array}\right]\left[\begin{array}{l}
\ddot{p}_{m}(t) \\
\ddot{q}_{m}(t)
\end{array}\right]+\left[\begin{array}{cc}
(\alpha m \pi)^{4} & 0 \\
0 & P_{\mathbf{K}}(m)
\end{array}\right]\left[\begin{array}{l}
p_{m}(t) \\
q_{m}(t)
\end{array}\right]+\left[\begin{array}{cc}
0 & -\beta^{2}(m \pi)^{2} \\
\beta^{2}(m \pi)^{2} & P_{\mathbf{D}}(m)
\end{array}\right]\left[\begin{array}{l}
\dot{p}_{m}(t) \\
\dot{q}_{m}(t)
\end{array}\right]=\left[\begin{array}{l}
0 \\
0
\end{array}\right]
$$

where the polynomials $P_{\mathbf{K}}(m)$ and $P_{\mathbf{D}}(m)$ are defined by Eq. (17).

Let us explicitly remark that the chosen boundary conditions and basis functions lead to a set of uncoupled evolution problems for each electromechanical pair of Fourier coefficients, hence it is possible to easily derive an analytical solution for the generic $m$-th pair.

Each set of equations for the Fourier coefficients $p_{m}$ and $q_{m}$ can be conveniently rewritten as:

$$
\left[\begin{array}{ll}
1 & 0 \\
0 & 1
\end{array}\right]\left[\begin{array}{l}
\ddot{p}_{m}(t) \\
\ddot{q}_{m}(t)
\end{array}\right]+\left[\begin{array}{cc}
\omega_{p}^{2}(m) & 0 \\
0 & \omega_{q}^{2}(m)
\end{array}\right]\left[\begin{array}{c}
p_{m}(t) \\
q_{m}(t)
\end{array}\right]+\left[\begin{array}{cc}
0 & -\sqrt{c(m)} \\
\sqrt{c(m)} 2 \zeta_{q}(m) \omega_{q}(m)
\end{array}\right]\left[\begin{array}{c}
\dot{p}_{m}(t) \\
\dot{q}_{m}(t)
\end{array}\right]=\left[\begin{array}{l}
0 \\
0
\end{array}\right]
$$

with

$$
\left\{\begin{array}{l}
\omega_{p}^{2}(m)=(\alpha m \pi)^{4} \\
\omega_{q}^{2}(m)=P_{\mathbf{K}}(m) \\
\zeta_{q}(m)=\frac{1}{2} \frac{P_{\mathbf{D}}(m)}{\sqrt{P_{\mathbf{K}}(m)}} \\
c(m)=(\beta m \pi)^{4}
\end{array} .\right.
$$




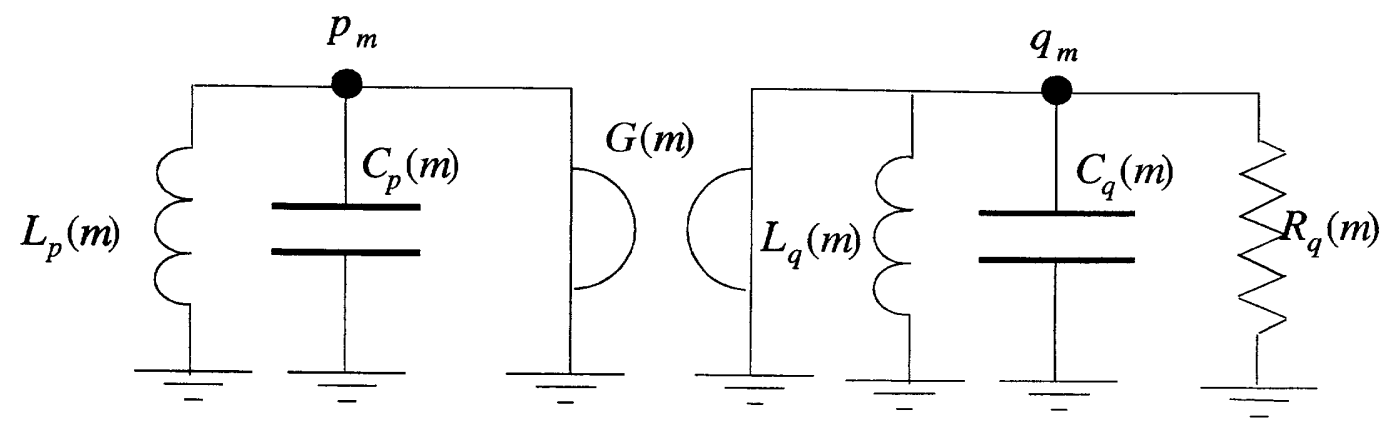

Fig. 4. Electric equivalent scheme of the coupled 2 DOF electromechanical system.

Let us remark that $\omega_{p}(m)$ and $\omega_{q}(m)$ are respectively the natural angular frequency of the mechanical and electrical $m$-th modes, while $c(m)$ denotes the $m$-th gyroscopic coupling coefficient and $\zeta_{q}(m)$ the damping factor of the $m$-th electrical mode.

\subsection{An electrical analog circuit for Eq. (19)}

From an electrical point of view, following [17], the set of ordinary differential Eq. (19), can be regarded as the governing equations of the two nodes circuit in Fig. 4, where the dimensionless electric components are given by:

$$
\left\{\begin{array}{l}
C_{p}(m)=C_{q}(m)=1 \\
L_{p}(m)=\frac{1}{\omega_{p}^{2}(m)} \\
L_{q}(m)=\frac{1}{\omega_{q}^{2}(m)} \\
R_{q}(m)=\frac{1}{2} \frac{1}{\zeta_{q}(m) \omega_{q}(m)} \\
G(m)=\sqrt{c(m)}
\end{array}\right.
$$

Thus, it is clear that the piezoelectric effect introduces a gyroscopic coupling (an electrical gyrator) between the $m$-th electrical and $m$-th mechanical modes; furthermore, because of the simply supporting conditions, the electrical and mechanical modal shapes coincide, and the evolution of the $m$-th pair of modes is completely independent of the one of a different pair of modes. The electric analog depicted in Fig. 4 represents a valuable tool when designing prototypes of Piezo-ElectroMechanical beams. Indeed, a purely electric representation of the whole electromechanical system allows for straightforward simulation of real electric networks by means of widely spread commercial codes (e.g. PSpiceAD, [18])

\subsection{Closed-loop interpretation of Eq. (19)}

A control engineer may view the electromechanical system in a different perspective. In particular for every pair of modes it is conceivable to define a mechanical system $S_{p}^{m}$ to be controlled and a passive 


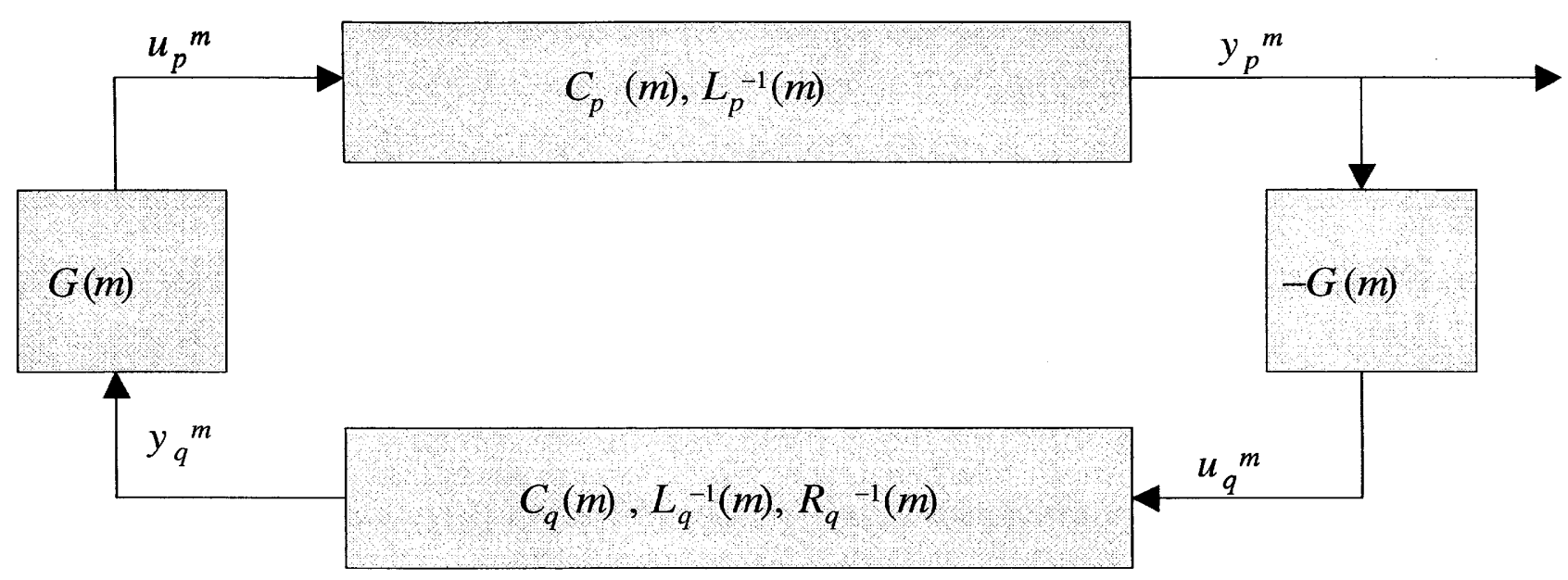

Fig. 5. Closed-loop intepretation of the piezoelectrically coupled electromechanical system.

electrical controller with second-order dynamics $S_{q}^{m}$ as:

system to be controlled $S_{p}^{m}: C_{p}(m) \ddot{p}_{m}(t)+\frac{1}{L_{p}(m)} p_{m}(t)=u_{p}^{m}(t), y_{p}^{m}(t)=\dot{p}_{m}(t)$,

passive controller $S_{q}^{m}: C_{q}(m) \ddot{q}_{m}(t)+\frac{1}{L_{q}(m)} q_{m}(t)+\frac{1}{R_{q}(m)} \dot{q}_{m}(t)=u_{q}^{m}(t), y_{q}^{m}(t)=\dot{q}_{m}(t)$,

where $\left(u_{p}^{m}, y_{p}^{m}\right)$, and $\left(u_{q}^{m}, y_{q}^{m}\right)$ are, respectively, the (input, measurement) of the mechanical and electrical systems.

Furthermore the following relations between the inputs and measurements of the coupled system hold:

$$
\left\{\begin{array}{l}
u_{p}^{m}(t)=G(m) y_{q}^{m}(t) \\
u_{q}^{m}(t)=-G(m) y_{p}^{m}(t)
\end{array} .\right.
$$

According to [19], the electrical system connected to the flexible structure is a controller having a second-order dynamics, introducing a velocity feedback.

The closed-loop poles of the system can be determined as the four zeros $s_{1}^{m}, \ldots s_{4}^{m}$ of the following polynomial:

$$
\begin{aligned}
P^{m}(s)= & s^{4}+2 \zeta_{q}(m) \omega_{q}(m) s^{3}+\left(\omega_{p}^{2}(m)+c(m)+\omega_{q}^{2}(m)\right) s^{2} \\
& +2 \zeta_{q}(m) \omega_{q}(m) \omega_{p}^{2}(m) s+\omega_{p}^{2}(m) \omega_{q}^{2}(m) .
\end{aligned}
$$

\section{Design of the passive optimal controller}

In this Section we design the optimal passive distributed controller following the pole placement technique applied to the evolution of each pair of electrical and mechanical modes. In particular, we determine an optimal expression for the stiffness and dissipative polynomials $P_{\mathbf{K}}(m)$ and $P_{\mathbf{D}}(m)$ appearing in the time evolution of the $m$-th electrical mode; consequently, we establish the forms of the operators $\mathbf{K}$ and $\mathbf{D}$. Once these operators are determined, an electrical circuit governed by a finite differences approximation of such operators is synthesized. 
The chosen optimality condition requires the determination of the values of $\omega_{q}(m)$ and $\zeta_{q}(m)$ in order to maximize the exponential time decay rate $\tau^{m}$ of the solution of the fourth order system Eq. (19), governing the time evolution of the $m$-th pair of electrical and mechanical modes, defined as:

$$
\tau^{m}=\min _{i=1, \ldots, 4}\left\{\left|\operatorname{Re}\left[s_{i}^{m}\right]\right|\right\}
$$

In order to maximize the exponential time decay $\tau^{m}$, the optimal values of $\omega_{q}(m)$ and $\zeta_{q}(m)$ are found by requiring the four roots of the closed loop polynomial $P^{m}(s)$ to be coincident (pole placement technique, see e.g. [1] and [20]).

Hence, we enforce the polynomial $P^{m}(s)$ to be factorized as:

$$
P^{m}(s) \equiv\left(s^{2}+2 \sigma(m) s+\left(\sigma(m)^{2}+\omega(m)^{2}\right)\right)^{2}
$$

Equating the coefficients of the above mentioned polynomials the following set of conditions is obtained:

$$
\left\{\begin{array}{l}
2 \zeta_{q}(m) \omega_{q}(m)=4 \sigma(m) \\
\left(\omega_{p}^{2}(m)+c(m)+\omega_{q}^{2}(m)\right)=6 \sigma(m)^{2}+2 \omega(m)^{2} \\
2 \zeta_{q}(m) \omega_{q}(m) \omega_{p}^{2}(m)=4 \sigma(m)^{3}+4 \sigma(m) \omega(m)^{2} \\
\omega_{p}^{2}(m) \omega_{q}^{2}(m)=\left(\sigma(m)^{2}+\omega(m)^{2}\right)
\end{array}\right.
$$

The previous set of relations imposes the following matching conditions on the electrical controller:

$$
\begin{aligned}
& \omega_{q}(m)=\omega_{p}(m), \\
& \zeta_{q}(m)=\frac{\sqrt{c(m)}}{\omega_{p}(m)} .
\end{aligned}
$$

Condition Eq. (22a) establishes that in order to maximize the time rate decay of the $m$-th pair of electrical and mechanical modes, the electrical $m$-th mode has to be resonant at the mechanical resonance frequency $\omega_{q}(m)$.

Thus, by the use of Eq. (20), the following conditions on the values of the polynomials $P_{\mathbf{K}}$ and $P_{\mathbf{D}}$ in correspondence with the mode number $m$ under control are stated:

$$
\left\{\begin{array}{l}
P_{\mathbf{K}}(m)=(\alpha m \pi)^{4} \\
P_{\mathbf{D}}(m)=2(\beta m \pi)^{2}
\end{array}\right.
$$

Consequently, from Eq. (21) the values of the real and imaginary part of the coincident roots are found to be:

$$
\left\{\begin{array}{l}
\sigma(m)=\frac{\sqrt{c(m)}}{2} \\
\omega(m)=\sqrt{\omega_{p}(m)^{2}-\frac{c(m)}{4}}
\end{array}\right.
$$


Hence, the damping ratio (defined as the sine of the phase of the coincident roots measured from the imaginary axis) becomes:

$$
\varsigma(m):=\frac{\sigma(m)}{\sqrt{\omega(m)^{2}+\sigma(m)^{2}}}=\frac{\sqrt{c(m)}}{2 \omega_{p}(m)} .
$$

Introducing the relations Eq. (20) into Eq. (24), we get the following expressions for the real and imaginary parts of the placed roots:

$$
\left\{\begin{array}{l}
\sigma(m)=\frac{(\beta m \pi)^{2}}{2} \\
\omega(m)=(m \pi)^{2} \sqrt{\alpha^{4}-\frac{\beta^{4}}{4}} .
\end{array}\right.
$$

Taking into account the definitions Eq. (18) of the parameters $\alpha$ and $\beta$, we get the following relations in terms of the properties of the piezo-electromechanical beam:

$$
\left\{\begin{array}{l}
\sigma(m)=\frac{1}{\omega_{0}} \frac{(m \pi)^{2}}{2} \frac{g_{\mathrm{hom}}}{l^{2}} \sqrt{\frac{1}{\gamma_{\mathrm{hom}} \rho_{\mathrm{hom}}}} \\
\omega(m)=\frac{1}{\omega_{0}}(m \pi)^{2} \frac{1}{l^{2}} \sqrt{\frac{k_{\mathrm{hom}}}{\rho_{\mathrm{hom}}}} \sqrt{\left(1-\frac{1}{4} \frac{g_{\mathrm{hom}}^{2}}{\gamma_{\mathrm{hom}} k_{\mathrm{hom}}}\right)}
\end{array}\right.
$$

and:

$$
\varsigma(m)=\frac{g_{\mathrm{hom}}}{2 \sqrt{\gamma_{\mathrm{hom}} k_{\mathrm{hom}}}} .
$$

Equation (25) clearly indicates that the optimal damping ratio is independent of the mode under control and is an increasing function of the electromechanical coupling coefficient $g_{\mathrm{hom}}$, and a decreasing function of the stiffness $k_{\text {hom }}$ and of capacitance per unit length $\gamma_{\text {hom }}$.

Conditions Eq. (23) provide the values of the $m$-th eigenvalues of the electrical eigenvalues for optimally damping the $m$-th mechanical mode. When one is concerned in damping out only one mechanical mode, for every choice of $A$ and $B$ in Eq. (16) it is possible to find constants $a_{2 i}$ and $b_{2 i}$ satisfying conditions Eq. (23). In order to simultaneously damp every mechanical mode, conditions Eq. (23) should be fulfilled for every choice of the index $m$. This may be accomplished by selecting the electrical operators as

$$
\left\{\begin{array}{l}
\mathbf{K}[\psi]=\alpha^{4} \psi^{I V} \\
\mathbf{D}[\dot{\psi}]=-2 \beta^{2} \dot{\psi}^{\prime \prime}
\end{array}\right.
$$

with domains:

$$
\begin{aligned}
\mathcal{D}(\mathbf{K})=\{ & f \in L^{2}: f, f^{\prime}, f^{\prime \prime}, f^{\prime \prime \prime} \text { are absolutely continuous, } \\
& \left.f^{I V} \in L^{2}, f(0)=f(1)=f^{\prime \prime}(0)=f^{\prime \prime}(1)=0\right\},
\end{aligned}
$$

and

$$
\mathcal{D}(\mathbf{D})=\left\{f \in L^{2}: f, f^{\prime} \text { are absolutely continuous, } f^{\prime \prime} \in L^{2}, f(0)=f(1)=0\right\} .
$$




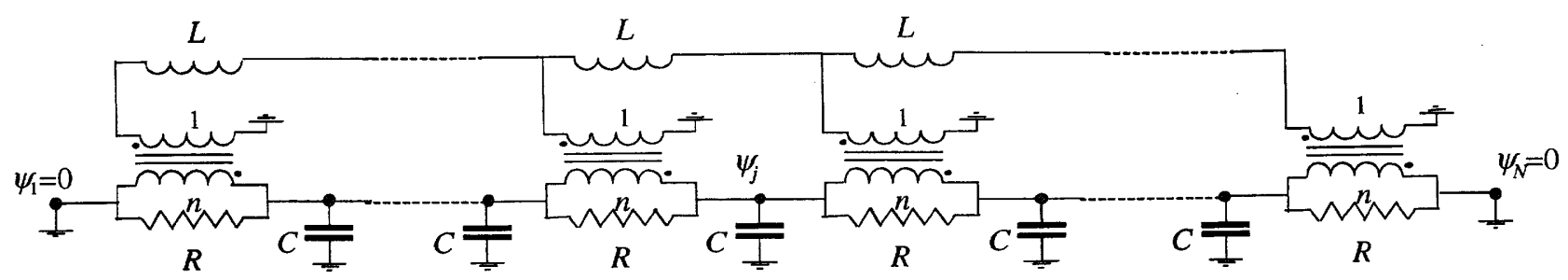

Fig. 6. Internal and external modules of the optimal electrical circuit interconnecting the bender transducers.

The optimal distributed passive circuit to be mechanically fed by the piezotransducers has to be governed by the following PDE:

$$
\ddot{\psi}+\mathbf{K}[\psi]+\mathbf{D}[\dot{\psi}]=0
$$

where the stiffness and damping operators are defined in Eq. (26). We remark that the self-adjoint operators $\mathbf{K}$ and $\mathbf{D}$ satisfy the relationship

$\mathbf{K} \propto \mathbf{D}^{2}$.

The found electrical circuit can be thought as the analog circuit of a simply supported vibrating beam, with an internal dissipation proportional to the curvature velocity $\dot{u}^{\prime \prime}$. The synthesis of a lumped electrical network governed by a finite differences approximation of Eq. (27) with Eq. (26) has been addressed in [8] and [9], leading to the circuit in Fig. 6.

The evolution equations for the free vibrations of the circuit in Fig. 6 are easily recognized to be:

$$
\left\{\begin{array}{l}
C \ddot{\psi}_{j}+\frac{1}{L n^{2}}\left(\psi_{j+2}-4 \psi_{j+1}+6 \psi_{j}-4 \psi_{j-1}+\psi_{j-2}\right)-\frac{1}{R}\left(\dot{\psi}_{j+1}-2 \dot{\psi}_{j}+\dot{\psi}_{j-1}\right)=0 \\
j=3, \ldots, N-2 \\
C \ddot{\psi}_{2}+\frac{1}{L n^{2}}\left(5 \psi_{2}-4 \psi_{3}+\psi_{4}\right)-\frac{1}{R}\left(\dot{\psi}_{3}-2 \dot{\psi}_{2}\right)=0 \\
C \ddot{\psi}_{N-1}+\frac{1}{L n^{2}}\left(5 \psi_{N-1}-4 \psi_{N-2}+\psi_{N-3}\right)-\frac{1}{R}\left(-2 \dot{\psi}_{N-1}+\dot{\psi}_{N-2}\right)=0 \\
\psi_{1}=0 \\
\psi_{N}=0
\end{array}\right.
$$

representing a central finite differences approximation of Eq. (27) with Eq. (26), if the used electrical elements satisfy the following set of conditions:

$$
\left\{\begin{array}{l}
C=k_{e e} \\
\frac{1}{L C n^{2}}\left(\frac{l}{N}\right)^{4}=\frac{k_{\mathrm{hom}}}{\rho_{\mathrm{hom}}} \\
\frac{1}{R C}\left(\frac{l}{N}\right)^{2}=2 g_{\mathrm{hom}} \sqrt{\frac{1}{\gamma_{\mathrm{hom}} \rho_{\mathrm{hom}}}}
\end{array} .\right.
$$

We explicitly remark that Eq. (30) establishes that the choice of the sole parameters $L$ and $R$ allows for multimodal vibration suppression. Nevertheless, Eq. (30) stem from the finite-differences approximation of the optimal operators in Eq. (26). Therefore the problem of the convergence of the solution of Eq. (29) to the corresponding solution of Eq. (27) is an important issue, as the self-resonance properties of considered PEM beam are greatly sensitive to small variations of constitutive parameters. In future, an 
accurate analysis of the lumped electrical network in Fig. 6 will be performed, addressing the problem of the convergence of the discrete circuit to the homogenized model and drawing comparisons between the natural frequencies and modal shapes predicted by the homogenized model and the ones achieved by the discrete system.

\section{Eigenvalue problem for the optimized PEM beam}

When the dissipative operator in Eq. (27) is discarded, a conservative PEM beam is obtained. Namely, the time evolution equations for the conservative simply supported PEM beam are:

$$
\left\{\begin{array}{l}
\ddot{u}+\alpha^{4} u^{I V}+\beta^{2} \dot{\psi}^{\prime \prime}=0 \\
\ddot{\psi}+\alpha^{4} \psi^{I V}-\beta^{2} \dot{u}^{\prime \prime}=0
\end{array},\right.
$$

where the following boundary conditions are imposed:

$$
\left\{\begin{array}{l}
u(0, t)=u(1, t)=u^{\prime \prime}(0, t)=u^{\prime \prime}(1, t)=0 \\
\psi(0, t)=\psi(1, t)=\psi^{\prime \prime}(0, t)=\psi^{\prime \prime}(1, t)=0
\end{array}\right.
$$

The electromechanical modal properties are obtained by looking for a solution of Eq. (31) together with the boundary conditions Eq. (32) in the form

$$
u(x, t)=m(x) \exp (\eta t), \quad \psi(x, t)=e(x) \exp (\eta t),
$$

where $m$ and $e$ indicate the electrical and mechanical parts of the electromechanical mode shape, and $\eta$ the eigenvalue. Therefore the following eigenvalue problem is obtained

$$
\left\{\begin{array}{c}
\eta^{2} m+\alpha^{4} m^{I V}+\eta \beta^{2} e^{\prime \prime}=0 \\
\eta^{2} e+\alpha^{4} e^{I V}-\eta \beta^{2} m^{\prime \prime}=0
\end{array}\right.
$$

with boundary conditions directly obtained from Eq. (32). By adapting the results obtained in [21] to the (generalized Sturm-Liouville) eigenvalue problem we obtain:

$$
\begin{array}{r}
\eta_{ \pm k}^{2}= \pm i \omega_{k}, \quad\left\{\begin{array}{c}
m_{ \pm k} \\
e_{ \pm k}
\end{array}\right\}=\left\{\begin{array}{c}
m_{k}^{R} \\
e_{k}^{R}
\end{array}\right\} \pm i\left\{\begin{array}{c}
m_{k}^{I} \\
e_{k}^{I}
\end{array}\right\}, \quad k=1,2, \ldots ; \\
\omega_{k} \in \mathrm{IR}^{+}, \quad\left\{\begin{array}{c}
m_{k}^{R} \\
e_{k}^{R}
\end{array}\right\},\left\{\begin{array}{c}
m_{k}^{I} \\
e_{k}^{I}
\end{array}\right\} \in \mathrm{IR}^{2} ;
\end{array}
$$

where $\omega_{k}$ and $\left\{\begin{array}{c}m_{ \pm k} \\ e_{ \pm k}\end{array}\right\}$ are the angular frequency and eigenvector of the $k$-th electromechanical mode of vibration of the distributed gyroscopic system, given by:

$$
\begin{aligned}
\omega_{k} & =\frac{1}{2}\left[\frac{1}{2}\left(k+\frac{\left(1-(-1)^{k}\right)}{2}\right)\right]^{2} \pi^{2} \beta^{2}\left((-1)^{k}+\sqrt{1+\left(\frac{2 \alpha^{2}}{\beta^{2}}\right)^{2}}\right), \\
\left\{\begin{array}{c}
m_{k}^{R} \\
e_{k}^{R}
\end{array}\right\} & =\left\{\begin{array}{l}
1 \\
0
\end{array}\right\} \sin \pi k x, \quad\left\{\begin{array}{c}
m_{k}^{I} \\
e_{k}^{I}
\end{array}\right\}=\left\{\begin{array}{c}
0 \\
(-1)^{k}
\end{array}\right\} \sin \pi k x, \quad k=1,2, \ldots
\end{aligned}
$$


Table 1

Properties and dimensions of the host beam

\begin{tabular}{ccc}
\hline Coefficient & Value & Units \\
\hline$l$ & $310^{-1}$ & {$[\mathrm{~m}]$} \\
$w$ & $210^{-2}$ & {$[\mathrm{~m}]$} \\
$h$ & $210^{-3}$ & {$[\mathrm{~m}]$} \\
$E_{b}$ & $7010^{9}$ & {$[\mathrm{~Pa}]$} \\
$\rho_{b}$ & 2700 & {$\left[\mathrm{~kg} \mathrm{~m}^{-3}\right]$} \\
\hline
\end{tabular}

Table 2

Properties and dimensions of the piezoelectric transducers (Piezo System T1 10-H4E-602, made of PSI$5 \mathrm{H} 4$ piecoceramic)

\begin{tabular}{ccc}
\hline Coefficient & Value & Units \\
\hline$\rho_{p}$ & 7800 & {$\left[\mathrm{~kg} \mathrm{~m}^{-3}\right]$} \\
$\left(s_{11}^{E}\right)^{-1}$ & $6210^{9}$ & {$[\mathrm{~Pa}]$} \\
$d_{31}$ & $-32010^{-12}$ & {$\left[\mathrm{~m} \mathrm{~V}^{-1}\right]$} \\
$\epsilon_{3}^{T}$ & $38008.8510^{-12}$ & {$\left[\mathrm{~F} \mathrm{~m}^{-1}\right]$} \\
$\delta$ & $2.6710^{-4}$ & {$[\mathrm{~m}]$} \\
$l_{p}$ & $2.510^{-2}$ & {$[\mathrm{~m}]$} \\
$d$ & $2.27310^{-3}$ & {$[\mathrm{~m}]$} \\
\hline
\end{tabular}

\section{Design of a prototype}

In order to assess the physical realizability and the efficiency of the proposed device, we consider an aluminum beam, the geometry of which is presented in Table 1 .

We position 10 bender transducers constituted by piezoceramic patches, from [22], made of lead zirconate titanate [PZT] (for more details on the different kinds of piezoelectric actuations see [23]). The characteristics of these piezoceramic transducers are listed in Table 2.

Thus, the covering factor can be estimated to be:

$$
c_{f}=91.67 \% \text {. }
$$

While, the capacitance and the coupling coefficient of the bender transducers are:

$$
\left\{\begin{array}{l}
k_{e e}=2 \frac{w\left(s_{11}^{E} \epsilon_{3}^{T}-d_{31}^{2}\right)}{s_{11}^{E} \delta} l_{p}=184.9 \mathrm{nF} \\
k_{m e}=\frac{h w d_{31}}{s_{11}^{E}}=1.43610^{-3} \mathrm{NmV}^{-1}
\end{array} .\right.
$$

The stiffness, coupling and mass per unit length of the homogenized PEM beam can be evaluated to be:

$$
\left\{\begin{array}{l}
k_{\mathrm{hom}}=\frac{E_{b} I_{b}+k_{m m}}{\left(1-c_{f}\right) \frac{E_{b} I_{b}+k_{m m}}{E_{b} I_{b}}+c_{f}}=1.926 \mathrm{Nm}^{2} \\
g_{\mathrm{hom}}=\frac{c_{f} k_{m e}}{\left(1-c_{f}\right) \frac{E_{b} I_{b}+k_{m m}}{E_{b} I_{b}}+c_{f}}=1.18910^{-3} \mathrm{NmV}^{-1} . \\
\rho_{\mathrm{hom}}=w h\left(\rho_{b}+2 c_{f} \frac{\delta}{h} \rho_{p}\right)=0.2462 \mathrm{~kg} \mathrm{~m}^{-1}
\end{array} .\right.
$$



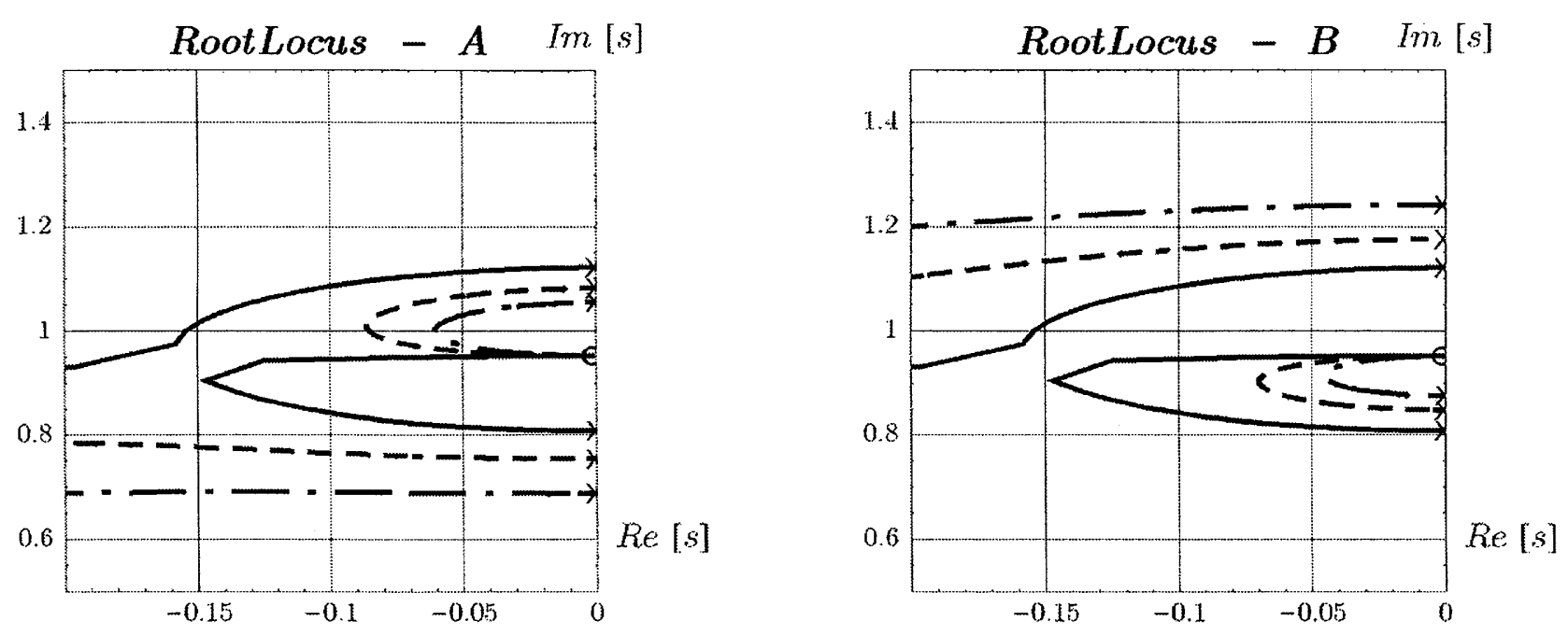

Fig. 7. Root loci of $P^{1}(s)$ for increasing values of $\zeta_{q}(1)$. Root Locus-A: dot-dash, $\omega_{q}(1)=0.8 \omega_{p}(1)$; dash, $\omega_{q}(1)=0.9 \omega_{p}(1)$; solid, $\omega_{q}(1)=\omega_{p}(1)$. Root Locus-B: dot-dash, $\omega_{q}(1)=1.2 \omega_{p}(1)$; dash, $\omega_{q}(1)=1.1 \omega_{p}(1)$; solid, $\omega_{q}(1)=\omega_{p}(1)$.

In the following, we choose as the frequency scaling parameter the angular frequency of the first mode of the simply supported beam without the piezoelectric patches:

$$
\omega_{0}=\frac{\pi^{2}}{l^{2}} \sqrt[4]{\frac{E_{b} I_{b}}{w h \rho_{b}}}=322.4 s^{-1}
$$

Hence the dimensionless parameters appearing in Eq. (18) can be numerically evaluated as

$$
\left\{\begin{array}{l}
\alpha=\frac{1}{l \sqrt{\omega_{0}}} \sqrt[4]{\frac{k_{\mathrm{hom}}}{\rho_{\mathrm{hom}}}}=3.105 \times 10^{-1} \\
\beta=\frac{1}{l} \sqrt{\frac{g_{\mathrm{hom}}}{\omega_{0}}} \sqrt[4]{\frac{1}{\gamma_{\mathrm{hom}} \rho_{\mathrm{hom}}}}=1.781 \times 10^{-1}
\end{array} .\right.
$$

From Eq. (30) it is easy to calculate the following values for the optimal resistance and inductance in the interconnecting network:

$$
L=0.5600 \mathrm{H}, \quad R=2643 \Omega,
$$

assuming unitary transformers $(n=1$ in Eq. (30)).

While from Eq. (25) the damping ratio of any mechanical mode of the simply supported PEM beam is determined to be:

$$
\varsigma(m)=16.46 \% \text {. }
$$

In Fig. 7 we plot the root locus of $P^{A}(s)$ for different values of the ratio $\frac{\omega_{p}(1)}{\omega_{q}(1)}$ as $\zeta_{q}(1)$ varies from 0 to $+\infty$.

From the exhibited plots it is clear that the matching condition Eq. (22a) is a mandatory requirement to obtain an efficient damping of mechanical vibration (high time rate decay). In fact, for a 10 per cent 
change of the ratio $\frac{\omega_{p}(1)}{\omega_{q}(1)}$ from the unity, the maximum achievable damping ratio dramatically decreases to one half.

As the proposed tuning procedure is simultaneously assuring the internal electromechanical resonance for every mode, and the root locus branch relative to the $m$-th mode is obtained by that relative to the first one by a homothetic transformation (which leaves the defined damping ratio unchanged), then the sensitivity argument developed for one mode holds for all the others. All previous considerations allow us to conclude that a multiresonance condition has to be achieved in order to guarantee an efficient broadband mechanical vibration damping.

\section{Conclusions}

In the present paper the concept of piezoelectric shunting by means of a single transducer suitably positioned on a structural member is generalized. Indeed we consider a beam hosting an array of $N$ piezoelectric transducers and look for a $N+1$ terminals electric network shunting them in an optimal way. If $N$ is sufficiently large and waves with wavelength reasonably greater than the size of the used transducers are considered, then we expect that the homogenized model for the controller may be suitable. In Section 3 such an infinite dimensional model is found, while in the subsequent sections its electric components are optimized to get the most efficient damping of mechanical vibrations. We have proved that, in principles, a multishape, multifrequency (i.e. a complete multimodal) vibration suppression is possible by means of a completely passive electric controller. This is obtained by synthesizing a distributed electric circuit governed by the same PDE governing the beam flexural vibrations.

However, the synthesis is achieved by the use of lumped circuital components, therefore the following problem arises: how efficient the finite dimensional circuit results are when compared with their homogenized counterpart. This is a crucial issue when prototypes of PEM beams must be designed, as sketched in the final section. Future works will be devoted to the comparison between the performances of homogenized electric controllers and their lumped approximations.

\section{Acknowledgments}

The partial support of the Engineering Science and Mechanics Department of Virginia Polytechnic Institute and State University is gratefully acknowledged by the authors. This presented research has been also partially supported by MIUR, Ministero per l'Innovazione, l'Università e la Ricerca Fondi Ricerca PRIN "Sintesi di circuiti piezoelettrici e tecniche di disaccoppiamento per il controllo di vibrazioni meccaniche" (protocollo 2001097882 _003) and by the Università di Roma "La Sapienza". The authors would also like to thank Prof. R. C. Batra, Corrado Maurini and Davide Spinello for useful discussions on the problem and for a detailed review of this work.

\section{References}

[1] N.W. Hagood and A. von Flotow, Damping of structural vibrations with piezoelectric materials and passive electrical networks, Journal of Sound and Vibrations 146 (1991), 243-268.

[2] J.J. Hollkamp, Multimodal passive vibration suppression with piezoelectric materials and resonant shunts, Journal of Intelligent Material Systems and Structures 5 (1994), 49-57. 
[3] A.J. Fleming, S. Behrens and S.O.R. Moheimani, Optimization and implementation of multimode piezoelectric shunt damping systems, IEEE/ASME Transactions on Mechatronics 7(1) (2002), 87-94.

[4] M. Kader, M. Lenczner and Z. Mrcarica, Distributed control based on distributed electronic circuits: application to vibration control, Microelectronics Reliability 41 (2001), 1857-1866.

[5] M. Bernadou and C. Haenel, Modelization and numerical analysis of active thin shell structures, Proceedings European Congress on Computational Methods in Applied Sciences and Engineering ECCOMAS, Barcelona, 2000.

[6] D. Halim and S.O.R. Moheimani, Experimental implementation of spatial $\mathcal{H}_{\infty}$ control on a piezoelectric-laminate beam, IEEE/ASME Transactions on Mechatronics 7(3) (2002), 346-356.

[7] K.-H. Hoffmann and N.D. Botkin, Homogenization of von Kármán plates excited by piezoelectric patches, ZAMM 80(9) (2000), 579-590.

[8] U. Andreaus, F. dell'Isola and M. Porfiri, Piezoelectric passive distributed controllers for beam flexural vibrations, Journal of Vibration and Control 10(5) (2004), 625-661.

[9] M. Porfiri, F. dell'Isola and F.M. Frattale Mascioli, Circuit analog of a beam and its application to multimodal vibration damping, using piezoelectric transducers, International Journal of Circuit Theory and Applications 32 (2004), 167-198.

[10] I. Chopra, Review of state of art of smart structures and integrated systems, AIAA Journal 40(11) (2002), 2145-2187.

[11] R.W. Newcomb, Linear Multiport Synthesis, New York: McGraw Hill, 1966.

[12] S. Vidoli and F. dell'Isola, Modal coupling in one-dimensional electromechanical structured continua, Acta Mechanica $141(2000), 37-50$.

[13] F. dell'Isola, C. Maurini and M. Porfiri, Passive damping of beam vibrations through distributed electric networks and piezoelectric transducers: prototype design and experimental validation, Smart Materials and Structures 13 (2004), 299-308.

[14] A.A. Samarskii and E.S. Nikolaev, Numerical Methods for Grid Equations, Birkhauser Verlag: Basel, 1989.

[15] R.F. Curtain and H.J. Zwart, An introduction to infinite-dimensional linear systems theory, Berlin: Springer-Verlag, 1995.

[16] C. Maurini, F. dell'Isola and D. del Vescovo, Comparison of Piezoelectronic networks acting as distributed vibration absorbers, Mechanical Systems and Signal Processing 18(5), 1243-1271.

[17] Y. Kagawa, T. Tsuchiya and N. Watatsuki, Equivalent circuit representation of a vibrating structure with piezoelectric transducers and stability consideration in the active damping control, Smart Materials and Structures 10 (2001), 389-394.

[18] Microsim PSpice A/D, Analog Digital Simulator, Microsim Corporation, 20 Fairbanks Irvine, CA 92718 USA, web site: http://www.microsim.com.

[19] J.N. Juang and M. Phan, Robust controller designs for second-order dynamic systems: A virtual passive approach, Journal of Guidance, Control and Dynamics 15(5) (1992), 1192-1198.

[20] D.W. Miller and E.F. Crawley, Theoretical and experimental investigation of space-realizable inertial actuation for passive and active structural control, Journal of Guidance, Control and Dynamics 11(5) (1988), 449-458.

[21] B. Yang, Eigenvalue inclusion principles for distributed gyroscopic systems, vibration analysis -analytical and computational, ASME, DE-37 (1991), 7-12.

[22] Piezo Systems, Inc, Cambridge, Massachussets, USA, web site: http://www.piezo.com.

[23] C. Niezreski, D. Brei, S. Balakrishnam and A. Moskalik, Piezoelectric actuation: State of the art, The Shock and Vibration Digest 33(4) (2001). 